\title{
Concentración de la tierra en Colombia
}

The Concentration of Land Ownership in Colombia

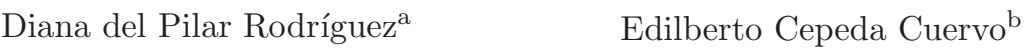 \\ dprodriguezc@unal.edu.co \\ ecepedac@unal.edu.co
}

\section{Resumen}

Este artículo incluye resultados del estudio de la concentración de la tierra en Colombia. Inicialmente, se consideran aspectos teóricos relacionados con la curva de Lorenz y el cálculo del Gini. Luego, se hace un análisis descriptivo de la concentración de la tierra, estableciendo la relación entre porcentajes de propietarios y porcentaje de tierra acumulada. Finalmente, se presentan los resultados del Gini, por regiones y por departamentos, para el año 2000.

Palabras clave: Curva de Lorenz, Colombia, concentracíón de la Tierra, Gini.

\begin{abstract}
This article includes results of the study of concentration of land ownership in Colombia. Initially, theoretical aspects are considered related to the Lorenz curve and Gini coefficient computation. Then, a descriptive analysis of the concentration of land ownership, establishing the relationship between owners percentages and percentage of accumulated land. Finally, we present the Gini results by region and department for the year 2000.
\end{abstract}

Key words: Colombia, Concentration of Land Ownership, Gini, Lorenz Curve.

\section{Introducción}

El nivel de concentración de variables económicas como ingreso, tierra y producción agrícola, se determina con los índices de Theil, Gini y Hirschman-Herfindal, entre otros. En este artículo se calcula la concentración de la tierra en Colombia utilizando el índice de Gini, y considerando únicamente los predios privados rurales (Sen 1986). En este estudio se excluyen los departamentos de Amazonas, Guainía,

\footnotetext{
${ }^{\text {a }}$ Profesor catedrático asociado. Universidad Distrital.

${ }^{\text {b}}$ Profesor asociado. Universidad Nacional de Colombia.
} 
Guaviare y Vichada, dado que, para estos departamentos, no se posee información de los predios registrados en el IGAC para los años entre 1996 y 2000.

La información catastral registrada por el Instituto Geográfico Agustín Codazzi (IGAC) para 1985 indica que el total de tierra registrada en Colombia era de 36.6 millones de hectáreas (Candelo et al. 2000). En 1996, según el IGAC y el Catastro de Antioquia, la tierra registrada era de 79.4 millones de hectáreas, presentándose un incremento del $217 \%$ en el número de hectáreas registradas, debido a la inclusión del departamento de Antioquia y de tierras de indígenas y de negritudes.

En 1996, el $52 \%$ de la tierra registrada era propiedad privada, mientras que en 1985 era del $89 \%$. Para 1996, de los 98.3 millones de hectáreas rurales registradas, el $32 \%$ corresponde a tierras de indígenas y de negritudes, y el $16 \%$ a tierras del Estado. En este año, el total de la tierra registrada correspondía al $45 \%$ de la superficie total de la República de Colombia, que es de 114 millones de hectáreas.

En el estudio de Candelo et al.(2000), publicado por la Contraloría General de la República, se afirma que hay una relación positiva entre la presencia de grupos alzados en armas y la concentración de la tierra. Identificando varias zonas del país que presentan este fenómeno, como el corredor de Frontino, que se inicia en el Urabá antioqueño y termina en Norte de Santander, pasando por el Magdalena bajo y medio, el pie de monte llanero, que comprende Arauca, Casanare, Meta, Putumayo y Caquetá, y en los departamentos del Valle del Cauca y Huila, los cuales tenían presencia paramilitar y/o guerrillera.

Según Machado (2004), la concentración en la propiedad de los factores productivos es el aspecto más notorio de la inequidad en el sector rural, generando serias implicaciones en el acceso y disponibilidad de recursos. Según Fajardo (2002), en 1996 los predios de 200 hectáreas correspondientes al $2.8 \%$ del total de las fincas, concentraban el $39.9 \%$ de la tierra, con un porcentaje mínimo de uso agrícola, y las fincas de hasta 5 hectáreas, que corresponden al $46.8 \%$ del total de los predios, sólo poseían el $3.2 \%$ de la tierra.

El informe del Banco Mundial (2004) afirma que en el año 2002 la desigualdad en la tenencia de la tierra es alta, con un índice de Gini de 0.85. Según este informe, esta desigualdad se debe a tres aspectos: el primero, la subutilización de la tierra productiva donde el suelo apto para la actividad agraria y zonas ecológicamente frágiles se usan en ganadería; el segundo, se refiere a la poca inversión de los gobiernos locales en el mejoramiento de los servicios públicos; y el tercero, incremento de la violencia en áreas con ausencia de oportunidades económicas, generando desplazamiento e inequidad social (Banco-Mundial \& CEDE 2004).

En este artículo, se aplican los conceptos de curva de Lorenz y de índice de Gini al estudio de la concentración de tierra en Colombia. El índice de Gini trata de poner en evidencia el mayor o menor grado de desigualdad en el reparto del total de los valores de la variable de interés. Es un indicador del grado de distribución de la variable. 
En la sección 2 se muestra la metodología para calcular la curva de Lorenz y el índice de Gini (Gini 1912). En la sección [3 se hace un estudio descriptivo de la distribución de la tierra en Colombia, considerando cada una de sus regiones geográficas. La sección 4, incluye un análisis de la distribución de la tierra en Colombia usando el índice de Gini. En la sección [5 se hacen algunas consideraciones finales y se dan algunas conclusiones relacionadas con los resultados encontrados en este estudio.

\section{Curva de Lorenz e índice de concentración de Gini}

Sea X una variable aleatoria con función de distribución $F(x)$, y media finita $\mu$. Dado

$$
F^{-1}(t)=\inf \{x: F(x) \geq t\}, \quad 0 \leq t \leq 1,
$$

la curva de Lorenz se define mediante una función $L(t)$, definida en el intervalo $[0,1]$, donde

$$
L(t)=\mu^{-1} \int_{0}^{F^{-1}(t)} y d F(y) .
$$

El índice de Gini, denotado IG, propuesto por Gini (1912), es una medida definida por

$$
I G=1-2 \int_{0}^{1} L(t) d F,
$$

cuya interpretación geométrica corresponde al doble del área comprendida entre la bisectriz del cuadrado unidad y la curva de Lorenz. Así,

$$
I G=2\left[1 / 2-\int_{0}^{1} L(t) d F\right]
$$

y $0 \leq I G \leq 1$, (Sen 1986). El índice de Gini se expresa comúnmente en porcentaje.

Para definir la curva de Lorenz y el índice de Gini, en el caso discreto se considera que $X$ es una variable aleatoria, con valores posibles $x_{1}<x_{2}<\ldots<x_{n}$, con $P\left(X=x_{i}\right)=p_{i}$ y media $\mu=\sum_{i=1}^{\infty} x_{i} p_{i}$. La curva de Lorenz es la función en $X$, definida a trozos, con vértices en $\left(F_{k}, G_{k}\right)$, donde $F_{k}=P\left\{X \leq x_{k}\right\}$ y $G_{k}=$ $\frac{1}{\mu} \sum_{i=1}^{k} x_{i} p_{i}$. Así, el área bajo la curva de Lorenz es $A=\sum_{k=1}^{n} A_{k}$, con $A_{k}=p_{k}\left(G_{k}+\right.$ $\left.G_{k-1}\right) / 2$ para $k=1,2, \ldots, n$ y $G_{0}=0$. (Mehran 1975, Gastwirth \& Glauberman 1976). Por tanto el índice de Gini es

$$
I G=1-2 A=1-\lim _{n \rightarrow \infty} \sum_{k=1}^{n} p_{k}\left(G_{k}+G_{k-1}\right)=1-E\left(G_{k}\right)-E\left(G_{k-1}\right) .
$$


equivalente a

$$
I G=1-\frac{1}{\mu} \sum_{k=1}^{\infty} x_{k} p_{k}^{2}-\frac{2}{\mu} \sum_{k=1}^{\infty} p_{k} \sum_{i=1}^{k-1} x_{i} p_{i}
$$

\subsection{Cálculo de la curva de Lorenz}

Para el estudio de la distribución de la tierra se debe tener en cuenta el concepto de distribución equitativa. Existe una distribución equitativa de la tierra entre los miembros de una población cuando a cada uno de ellos le corresponde una fracción proporcional del total de la misma. Por ejemplo, en una población de 100 propietarios, si la tierra está distribuida equitativamente, cada uno de los miembros de la población debe ser propietario de un $1 \%$ del total de la superficie de la tierra.

La función de equidistribución está determinada por la ecuación $G(x)=x$, donde $x$ es el porcentaje de los propietarios de la tierra y $y=G(x)$ es el porcentaje de tierra que le corresponde. Cuando existe concentración de la tierra, la curva de Lorenz se encuentra por debajo de la diagonal, y a mayor concentración de la tierra mayor es la curvatura.

La metodología para construir la curva de Lorenz es la siguiente:

1. Ordenar la información del área de los predios en sentido ascendente, en hectáreas, y establecer el número de rangos.

2. Calcular las frecuencias acumuladas del número de hectáreas y del número de propietarios, de acuerdo con los rangos establecidos.

3. Determinar y localizar en el plano coordenado los pares ordenados $(x, y)$, donde $x$ corresponde al porcentaje acumulado de propietarios y $y$ al porcentaje acumulado de hectáreas.

4. La curva de Lorenz se obtiene uniendo los puntos correspondientes a los pares ordenados $(x, y)$, mediante una línea continua.

\subsection{Cálculo del Gini}

El coeficiente Gini es el cociente entre el área de la región limitada por la curva de Lorenz y la recta $\mathrm{Y}=\mathrm{X}$, y el área de la región limitada por las rectas $\mathrm{X}=1, \mathrm{Y}=\mathrm{X}$ y $\mathrm{Y}=0$. Así,

$$
\begin{aligned}
I G & =2\left(\frac{1}{2}-T\right) \\
& =1-2 T,
\end{aligned}
$$

donde $T$ es el área comprendida entre la curva de Lorenz, el eje $X$ y $X=1$. 
Para calcular el área bajo la curva de Lorenz, T, se realiza la suma de las áreas de los trapecios cuyas bases corresponden a los rangos porcentuales acumulados de los propietarios de la tierra. En consecuencia,

$$
T=\frac{1}{2} \sum_{i=1}^{n}\left[G_{i-1}(x)+G_{i}(x)\right]\left[P\left(X \leq x_{i}\right)-P\left(X \leq x_{i-1}\right)\right],
$$

donde $G_{i}, i=1,2, \ldots, n$, es el porcentaje acumulado de superficie de tierra rural y $P\left(X \leq x_{i}\right), i=1,2, \ldots, n$, es el porcentaje acumulado de propietarios de la tierra. Este método de cálculo del Gini se considera bastante preciso cuando se aplican a distribuciones de ocho o más rangos (Lora 1991).

\section{Distribución de tierra en Colombia}

\subsection{Naturaleza de los datos}

La información de predios rurales registrados fue suministrada por el Instituto Geográfico Agustín Codazzi (IGAC) y para el año de 1996 por el catastro de Antioquia. Los periodos de tenencia de la tierra con registro catastral son los años 1986, 1996, 1999, 2000 y 2001. Esta información está desagregada por cada municipio del país, e incluye las siguientes variables: código del departamento, código del municipio (este código es el mismo que utiliza el DANE), índice del rango, nombre del departamento, nombre del municipio, rangos de área en hectáreas, total de predios por rango, total de propietarios por rango, total de superficie por rangos.

En la tabla 1 se muestra la estructura de la base de datos presentada por municipios. Se consideran 13 rangos, en hectáreas, como es registrado por el IGAC, tomando como ejemplo el municipio de Sopó en el departamento de Cundinamarca. Estos rangos en hectáreas fueron asumidos por el DANE y el IGAC para estudios de avalúo.

\subsection{Distribución de la tierra}

En esta sección se presentan datos correspondientes a la estructura de concentración de la tierra en Colombia, para los años 1996 y 2000. La tabla 2 muestra datos de la concentración de tierra en la región andina. Indica, por ejemplo, que en 1996, en el departamento de Boyacá, el $45.1 \%$ de la tierra rural estaba distribuida en el $95 \%$ de los propietarios. Así, el $54.9 \%$ de la tierra rural estaba distribuida en un $5 \%$ de los propietarios rurales. Para este departamento, en el año 2000 se evidencia un incremento en la concentración de la tierra, debido a que el porcentaje de la tierra rural distribuida en el $5 \%$ de los propietarios alcanza el $58.3 \%$. En la misma tabla, se muestra que en Quindío, en este periodo de tiempo, el porcentaje de tierra en manos del $95 \%$ de los propietarios pasó del $20 \%$ al $44.6 \%$, indicando 
un proceso de desconcentración. Este fenómeno también se presenta en el Huila, que es el departamento de la región andina con menor concentración.

\begin{tabular}{crrrrr}
\hline Rango (Has) & Predios & Propietarios & Área & Constr. $\left(\mathrm{m}^{2}\right)$ & Avalúo \\
\hline$<$ a 1 & 73 & 80 & 215.118 & 4.809 & 31.6893 .500 \\
$1-3$ & 48 & 93 & 900.477 & 7.522 & 105.429 .700 \\
$3-5$ & 23 & 27 & 887.665 & 2.468 & 334.404 .000 \\
$5-10$ & 16 & 19 & 1.201 .487 & 15.000 & 1.491 .322 .000 \\
$10-15$ & 22 & 25 & 2.692 .937 & 2.234 & 588.022 .000 \\
$15-20$ & 9 & 10 & 1.563 .713 & 6.128 & 671.727 .000 \\
$20-50$ & 30 & 39 & 9.784 .236 & 24.262 & 3.939 .730 .000 \\
$50-100$ & 6 & 11 & 4.147 .000 & 4.898 & 1.078 .234 .000 \\
$100-200$ & 8 & 9 & 10.713 .998 & 14.765 & 2.208 .735 .000 \\
$200-500$ & 2 & 2 & 6.800 .000 & 2.680 & 1.958 .803 .000 \\
$500-1000$ & 0 & 0 & 0 & 0 & 0 \\
$1000-2000$ & 1 & 1 & 10.710 .000 & 0 & 369.500 .000 \\
$>2000$ & 0 & 0 & 0 & 0 & 0 \\
\hline
\end{tabular}

Tabla 1: Distribución de la tierra por rangos

\begin{tabular}{l|ccccc|ccccc}
\hline \% de Prop. & 40 & 60 & 80 & 90 & 95 & 40 & 60 & 80 & 90 & 95 \\
\hline Antioquia & 1.8 & 3.5 & 13.3 & 32.9 & 38.6 & 1.2 & 3.5 & 10.1 & 20.0 & 39.4 \\
Boyacá & 3.4 & 7.9 & 16.1 & 30.0 & 45.1 & 3.2 & 8.1 & 18.1 & 30.0 & 41.7 \\
Caldas & 1.5 & 4.7 & 18.0 & 29.2 & 45.6 & 3.1 & 7.2 & 17.8 & 30.1 & 46.8 \\
Cund. & 2.8 & 7.0 & 20.0 & 30.7 & 43.7 & 3.0 & 9.1 & 20.4 & 33.0 & 46.1 \\
Huila & 3.1 & 9.0 & 21.1 & 37.5 & 51.6 & 3.2 & 10.2 & 26.6 & 45.2 & 62.0 \\
N. Sant. & 2.6 & 8.5 & 22.2 & 35.3 & 47.1 & 3.1 & 10.0 & 28.9 & 45.1 & 59.2 \\
Quindío & 1.9 & 4.5 & 11.3 & 19.5 & 26.6 & 2.9 & 7.9 & 19.0 & 31.1 & 44.6 \\
Risaralda & 2.4 & 7.6 & 17.9 & 28.1 & 40.0 & 3.3 & 9.0 & 20.2 & 32.0 & 48.0 \\
Santander & 2.0 & 8.1 & 18.8 & 32.1 & 44.0 & 3.3 & 8.1 & 20.1 & 37.0 & 49.1 \\
Tolima & 2.2 & 8.0 & 20.1 & 38.3 & 49.1 & 3.0 & 9.2 & 22.1 & 40.0 & 57.1 \\
\hline
\end{tabular}

Tabla 2: Porcentaje de tenencia de tierra, región andina(1996 a 2000)

En la región atlántica se presenta desconcentración en la tenencia de la tierra rural en el periodo comprendido entre 1996 y 2000, como se observa en la tabla 3. En todos los departamentos disminuyó la cantidad de tierra en manos del $5 \%$ de los propietarios. Durante este periodo, en el departamento del Cesar, que es donde se presentan los mayores cambios, el porcentaje de la tierra en manos del $5 \%$ de los propietarios pasó del $50.8 \%$ al $33.4 \%$, presentándose un cambio del $17.4 \%$ en la concentración de la tierra. La mayor concentración de tierra, en el año 2000, se presenta en el departamento de La Guajira con el $55.6 \%$ de la tierra en manos del $95 \%$ de los propietarios. 


\begin{tabular}{l|ccccc|ccccc}
\hline \% de Prop. & 40 & 60 & 80 & 90 & 95 & 40 & 60 & 80 & 90 & 95 \\
\hline Atlántico & 4.5 & 12.6 & 29.0 & 45.0 & 54.3 & 4.1 & 12.1 & 30.0 & 46.0 & 66.1 \\
Bolívar & 4.3 & 11.1 & 28.0 & 40.0 & 52.0 & 4.1 & 8.7 & 27.8 & 47.2 & 64.3 \\
Cesar & 3.6 & 11.0 & 24.1 & 38.5 & 49.2 & 5.2 & 16.3 & 34.3 & 52.5 & 66.6 \\
Córdoba & 1.9 & 9.1 & 23.0 & 39.0 & 54.4 & 2.3 & 8.5 & 25.3 & 40.0 & 60.0 \\
Guajira & 1.0 & 3.4 & 9.2 & 14.5 & 20.0 & 3.1 & 10.5 & 25.8 & 43.1 & 55.6 \\
Magdalena & 3.0 & 12.0 & 26.5 & 38.9 & 52.2 & 3.7 & 14.1 & 30.3 & 46.8 & 65.4 \\
Sucre & 1.9 & 7.6 & 25.4 & 40.1 & 57.5 & 2.3 & 10.0 & 27.8 & 48.3 & 63.5 \\
\hline
\end{tabular}

Tabla 3: Porcentaje de tenencia de tierra, región atlántica(1996 a 2000)

La región pacífica muestra mayor concentración que las regiones atlántica y andina. La tabla 4 muestra que el departamento de Chocó tiene un cambio notorio en la estructura de tenencia de la tierra, debido al incremento de predios registrados durante el periodo 1996 a 2000. En el año 2000, en el departamento del Valle del Cauca, el $64.3 \%$ de la tierra estaba en manos del $5 \%$ de los propietarios y, en el departamento del Cauca, el $61.5 \%$ de la tierra correspondía al $5 \%$ de los propietarios.

\begin{tabular}{l|ccccc|ccccc}
\hline \% de Prop. & 40 & 60 & 80 & 90 & 95 & 40 & 60 & 80 & 90 & 95 \\
\hline Cauca & 1.3 & 4.6 & 12.5 & 20. & 30. & 2.0 & 6.9 & 18.3 & 28.7 & 38.5 \\
Chocó & 0.06 & 0.9 & 4.5 & 5.2 & 7.1 & 0.03 & 14.2 & 38.5 & 56.1 & 66.0 \\
Nariño & 2.4 & 8.2 & 16.9 & 28.6 & 35.7 & 2.9 & 9.3 & 19.2 & 32.1 & 42.5 \\
Valle & 0.8 & 3.1 & 11.1 & 22.9 & 31.8 & 0.7 & 3.4 & 11.9 & 24.2 & 35.7 \\
\hline
\end{tabular}

Tabla 4: Porcentaje de tenencia de tierra, región pacífica(1996 a 2000)

En la región orinoquía-amazonía se encuentran varios de los departamentos con mayor concentración de tierra de Colombia. Por ejemplo, para el año 2000, en el departamento de Arauca, el $33 \%$ de la tierra estaba en manos del $95 \%$ de los propietarios, mientras que en los departamentos de Putumayo, Casanare y Meta, estaba el $35.7 \%$, 37.6\% y el $38.5 \%$, respectivamente, como se muestra en la tabla 5

\begin{tabular}{l|ccccc|ccccc}
\hline \% de Prop. & 40 & 60 & 80 & 90 & 95 & 40 & 60 & 80 & 90 & 95 \\
\hline Caquetá & 1.6 & 5.1 & 10.4 & 15.6. & 18.1 & 7.5 & 14.5 & 29.3 & 39.6 & 48.0 \\
Meta & 0.9 & 3.1 & 10.0 & 17.6 & 28.1 & 0.9 & 3.0 & 10.2 & 20.7 & 38.0 \\
Arauca & 3.1 & 8.0 & 16.1 & 22.1 & 33.2 & 2.6 & 8.0 & 15.6 & 22.1 & 33.2 \\
Casanare & 1.2 & 5.1 & 12.9 & 21.9 & 34.4 & 1.4 & 7.5 & 14.5 & 26.7 & 37.6 \\
Putumayo & 0.8 & 3.1 & 11.1 & 22.9 & 31.8 & 0.7 & 3.4 & 11.9 & 24.2 & 35.7 \\
\hline
\end{tabular}

Tabla 5: Porcentaje de tenencia de tierra, región orinoquía y amazonía(1996 a 2000)

Las figuras 1, 2] y 3 muestran la evolución de la estructura de concentración de la tierra en Colombia, para el $60 \%$, $90 \%$ y $95 \%$ de los propietarios, mostrando 
de nuevo la mayor concentración en Antioquia y Boyacá en la región andina; en los departamentos de Cauca y Valle del Cauca en la región pacífica, en los departamentos del pie de monte llanero (Meta, Casanare, Arauca y una menor concentración en Caquetá y Putumayo). Por otra parte, en la región atlántica, que es la de menor concentración, los departamentos con mayor desigualdad en la tenencia de la tierra son Córdoba y Guajira.

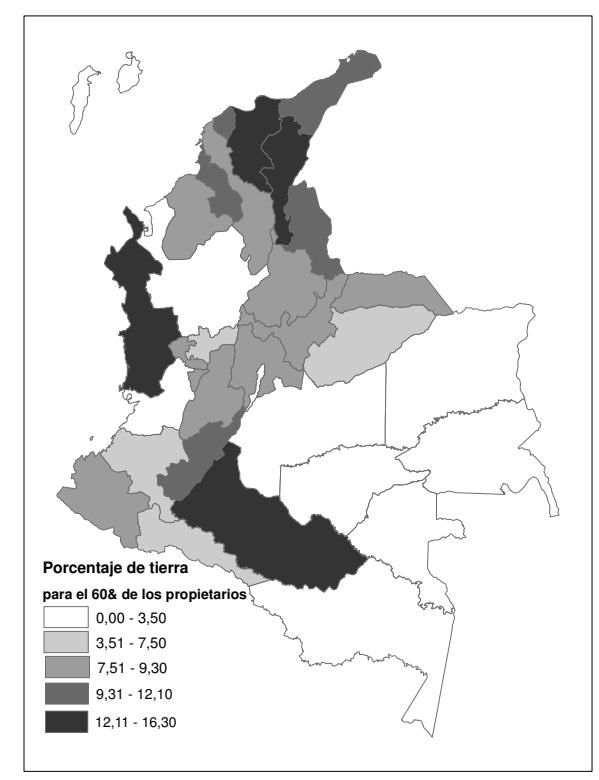

Figura 1: Distribución del $60 \%$ de la tierra en Colombia(2000)

\section{Análisis da la distribución de la tierra usando el índice de Gini}

\subsection{Curva de Lorenz}

La estructura de tenencia de la tierra, en este caso, es especificada mediante la curva de Lorenz. Esta curva muestra la proporción de tenencia de la tierra como una función de la proporción de propietarios. La figura 4 muestra la curva de Lorenz para la distribución de la tierra en Colombia y en los departamentos de Antioquia, Córdoba y Cesar, en el año 2000. En esta figura, se observa que la mayor concentración de la tierra se presentó en el departamentos de Antioquia, pues la concavidad de la curva de Lorenz es mayor con respecto a los departamentos de Córdoba y Cesar. Si comparamos la curva de Lorenz para Colombia con la 


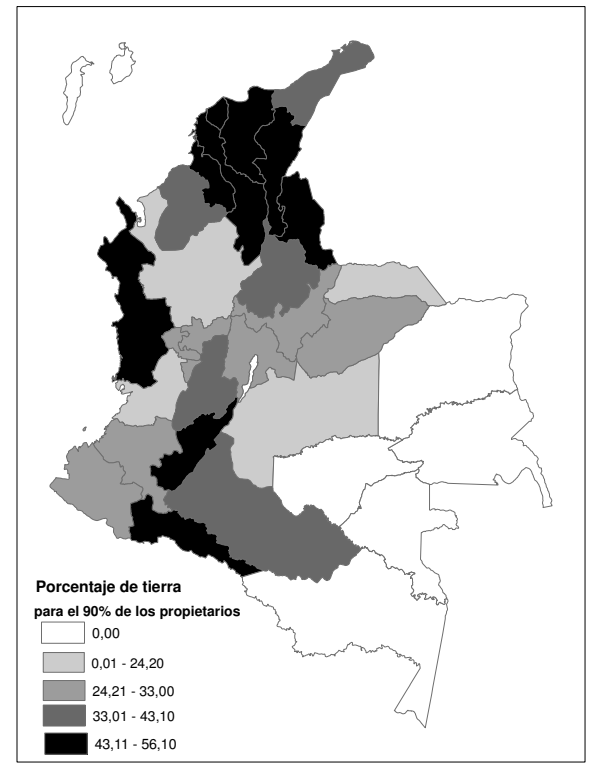

Figura 2: Distribución del $90 \%$ de la tierra en Colombia(2000)

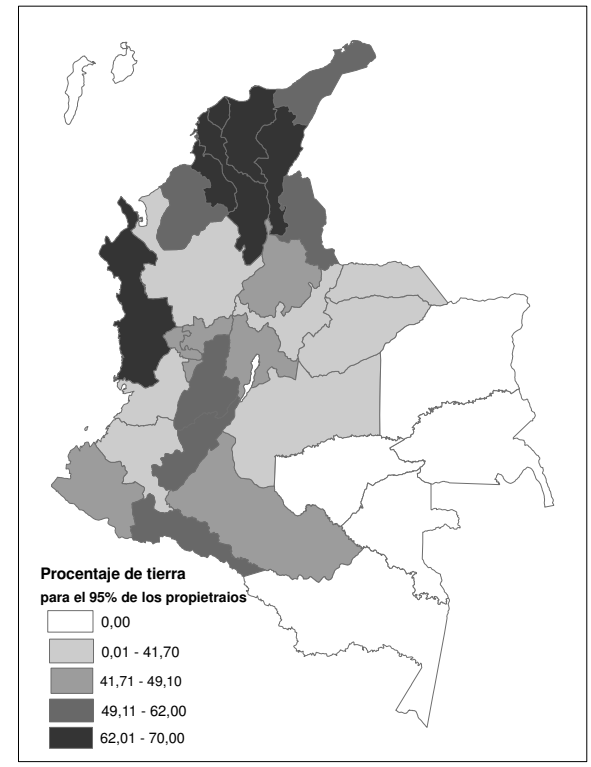

Figura 3: Distribución del $95 \%$ de la tierra en Colombia

Comunicaciones en Estadística, junio 2011, Vol. 4, No. 1 
curva del departamento de Antioquia sus concavidades son similares, lo que indica similaridad en la estructura de concentración de la tierra.

La estructura de tenencia de la tierra en Colombia para el año 2000 muestra que el $57.1 \%$ de los propietarios tiene el $3 \%$ de la tierra, y el $97.1 \%$ de los propietarios poseen el 41,6\% de la tierra; es decir, el $2.9 \%$ de los propietarios de fincas en el país poseen el $58.4 \%$ de la tierra registrada (ver Figura 4).
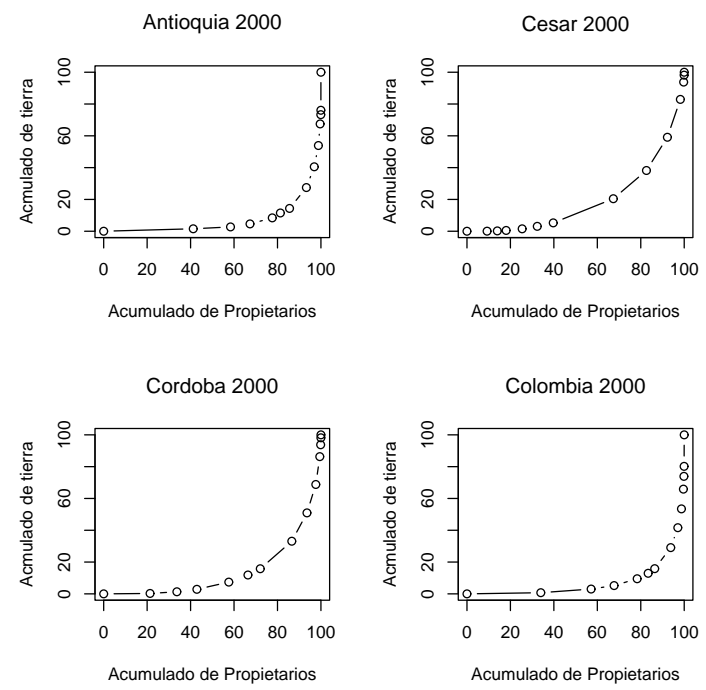

Figura 4: Curva de Lorenz año 2000.

\subsection{Cálculo del Gini para el año 2000}

El Gini para la concentración de la tierra rural en Colombia, en el año 2000, es de 0.8479. Esto indica una alta acumulación de la tierra en pocos propietarios. Como se observa en la tabla 6. la región con más alta concentración de la tierra es orinoquía - amazonía con un Gini de 0.7843 y la menor es la región atlántica con un Gini de 0.6811.

\begin{tabular}{l|c}
\hline Región & Gini \\
\hline Andina & 0.7579 \\
Atlántica & 0.6811 \\
Pacífica & 0.7584 \\
Orinoquía - Amazonía & 0.7843 \\
\hline
\end{tabular}

Tabla 6: Gini por regiones, año 2000.

En la región atlántica, se encuentra Cesar, que es el departamento con menor concentración de tierra en el país en el año 2000, con un Gini de 0.6178. En esta 
región, el departamento donde se presenta una mayor concentración es Córdoba, con un Gini de 0.7295.

La región andina tiene un Gini de 0.7579. Allí se encuentra el departamento que presenta la mayor concentración de tierra del territorio colombiano, Antioquia, con un Gini de 0.8583. El departamento con menor concentración de tierra en esta región es Norte de Santander, con un Gini de 0.6923. La tabla 7 muestra el Gini para 27 de los departamentos del país. La alta concentración de la tierra en el departamento de Antioquia también es evidenciada en el estudio de Gaviria y Muñoz (2007), que muestra la relación entre propiedad de la tierra y desplazamiento forzado, sustentada en datos que demuestran cómo a medida que aumenta el número de personas desplazadas, aumenta la concentración de la propiedad de la tierra, con los consecuentes efectos negativos sobre la producción agrícola y la población rural.

\begin{tabular}{lc|lc}
\hline Departamento & Gini & Departamento & Gini \\
\hline Antioquia & 0.8583 & Guajira & 0.7090 \\
Boyacá & 0.7619 & Magdalena & 0.6547 \\
Caldas & 0.7821 & Sucre & 0.6972 \\
Cundin. & 0.7529 & Cauca & 0.7579 \\
Huila & 0.7025 & Chocó & 0.6314 \\
Nort. San. & 0.6923 & Nariño & 0.7646 \\
Quindío & 0.7755 & Valle & 0.8429 \\
Risaralda & 0.7591 & Casanare & 0.8108 \\
Santander & 0.7539 & Caquetá & 0.6904 \\
Tolima & 0.7404 & Meta & 0.8544 \\
Atlántico & 0.6662 & Arauca & 0.8198 \\
Bolívar & 0.6937 & Casanare & 0.8108 \\
Cesar & 0.6178 & Putumayo & 0.7459 \\
Córdoba & 0.7295 & & \\
\hline
\end{tabular}

Tabla 7: Gini por departamentos (2000)

La región pacífica tiene, para este año, un Gini de 0.7584. Allí se encuentra el departamento del Valle del Cauca, el tercero con mayor concentración en el país. $\mathrm{Su}$ Gini es de 0.8429. En esta región el departamento con menos concentración de tierras es Chocó, con un Gini de 0.6314.

La región con mayor concentración de tierras es orinoquía-amazonía, con un Gini de 0.7843. Allí se encuentran algunos de los departamentos con mayor concentración de tierras en Colombia: Meta, que tiene la segunda concentración de tierras más alta del país, con un Gini de 0.8544, Arauca con un índice de 0.8198 y Casanare con 0.8108. El departamento con menor concentración es Caquetá, con un Gini de 0.6904. En esta región la alta concentración se debe, según Ortiz (2003), a la presencia de un alto porcentaje de cultivos ilícitos y de actores del conflicto armado en Colombia: paramilitares, guerrilla y fuerza pública. Igualmente, la presencia de narcotráfico y su relación con el conflicto armado, genera desplazamiento 
y, posiblemente, mayor concentración de tierra (Díaz \& Sánchez 2004).

En la figura 5 se representa el índice de Gini por departamentos. En ella se observa que los departamentos del pie de monte llanero, Antioquia y Valle del Cauca, son los que tienen mayor concentración de tierra; los de menor concentración están en la región atlántica.

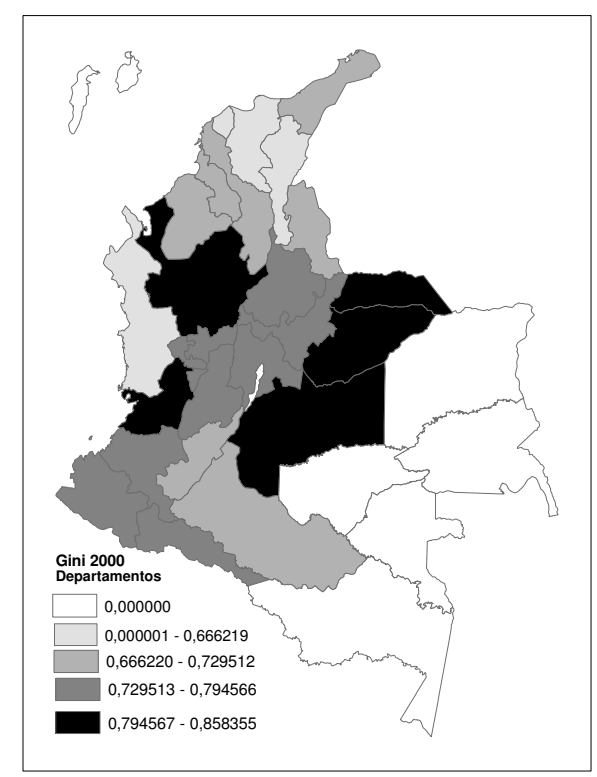

Figura 5: Gini - 2000 por departamentos

\section{Conclusiones}

Colombia presenta un alto nivel de concentración de tierras, con un índice de Gini de 0.8479. Los departamentos del pie de monte llanero, Antioquia y Valle del Cauca, son los que tienen mayor concentración. En Colombia el $80 \%$ de la tierra está en manos del $10 \%$ de los propietarios. En el departamento del Valle del Cauca el $80 \%$ de la tierra está en manos del $11.5 \%$ de los propietarios, y en Antioquia el $80 \%$ de la tierra está en manos del $11 \%$ de los propietarios. Este alto grado de concentración de tierras se presenta, en general, en todos los departamentos y regiones del país.

La concentración de tierras, en Colombia, tiene múltiples causas. Ibañez \& Querubín (2004) afirma que una de las causas de alta concentración está dada por la prolongación de los conflictos agrarios del siglo pasado, y el narcotráfico, que tienen una relación estrecha con el conflicto armado debido a la lógica e intereses de 
los actores (paramilitares, guerrilla y fuerza pública), generando desplazamiento forzoso (Díaz \& Sánchez 2004, Ortiz 2003).

Dada la distribución de los indicadores de concentración de la tierra en Colombia, podemos concluir, como en el estudio de Gaviria \& Muñoz (2007), que los incrementos en la concentración de la tierra parecen estar relacionados con el conflicto armado y, en especial, con los móviles de intimidación a la población no partícipe, que ha traído consigo un aumento significativo de personas que abandonan sus tierras por causa de la violencia (Ibañez \& Querubín 2004).

El estudio de los incrementos en la concentración de la tierra y en el desplazamiento forzado, resultado de luchas históricas por el poder y la acumulación de la riqueza (Gaviria \& Muñoz 2007), son áreas de especial interés para el desarrollo de investigaciones futuras que permitan entender los diversos fenómenos sociales y políticos presentes en Colombia durante los últimos años.

\section{Recibido: 27 de octubre de 2010} Aceptado: 31 de enero de 2011

\section{Referencias}

Atkinson, A. (1983), The Economics of Inequality, Oxford: Clarendon Press.

Banco-Mundial \& CEDE (2004), 'Colombia: una política de tierras en transición', Documento CEDE 2004-29 29, 1-100.

Candelo, R., Mera, D. \& Ossa, C. (2000), 'Propiedad rural y reforma agraria en Colombia: un debate sobre el periodo 1985-1996', Economía Colombiana y Coyuntura Económica 278, 23-32.

Díaz, A. \& Sánchez, F. (2004), 'Geografía de los cultivos ilícitos y conflicto armado en Colombia', Documento CEDE. (Edición Electrónica) 18, 1-78.

Fajardo, D. (2002), 'Notas para la formulación de un programa de desarrollo rural para la paz', Colombia tierra y paz .

Fernández, A. \& Costa, M. (1998), 'Descomposición de los índices de Gini y entropía generalizada: desigualdad y nivel de estudios en España e Italia (1991)', Estadística Española 40, 233-256.

Gastwirth, J. \& Glauberman, M. (1976), 'The Interpolation of the Lorenz Curve and Gini Index from Grouped Data', Econometrica 44, 479-483.

Gastwirth, J. L. (1971), 'A General Definition of the Lorenz Curve', Econometrica 39, 1037-1039.

Gaviria, C. \& Muñoz, J. (2007), 'Desplazamiento forzado y propiedad de la tierra en Antioquia, 1996-2004', Universidad de Antioquia-Lecturas de Economía 66, 9-46. 
Gini, C. (1912), Variabilita e Mutabilita, Bologna: Tipografia di Paolo Cuppini.

Gini, C. (1921), 'Measurement of Inequality of Income', The Economic Journal 31, 124-126.

Ibañez, A. \& Querubín, P. (2004), 'Acceso a tierras y desplazamiento forzado en Colombia', Documento CEDE 23, 1-114.

Lora, E. (1991), Técnicas de Medición Económica, TM editores Fedesarrollo.

Machado, A. (2004), La Academia y el Sector Rural, Unibiblos.

Medina, F. (2001), 'Consideraciones sobre el índice de Gini para medir la concentración del ingreso', Naciones Unidas. Estudios estadíticos y prospectivos 30, 15-22.

Mehran, F. (1975), 'Bounds on Gini Index Based on Observed Points Of Lorenz Curve', Journal of American Statistical Association 70, 64-66.

Ortiz, C. (2003), 'Cultivos ilícitos y nueva ruralidad en Colombia', Cuadernos de Desarrollo Rural 50, 115-165.

R Development Core Team (2007), R: A Language and Environment for Statistical Computing, R Foundation for Statistical Computing, Vienna, Austria. ISBN 3-900051-07-0.

*http://www.R-project.org

Sarabia, J., Castillo, E., Pascual, M. \& Slottje, D. (2005), 'Mixture Lorenz curves', Economics Letters 89, 89-94.

Sarabia, J., Castillo, E. \& Slottje, D. (1999), 'An Ordered Family of Lorenz Curves', Journal of Econometrics 91, 43-60.

Sarabia, J., Castillo, E. \& Slottje, D. (2001), 'An exponential family of Lorenz curves', Journal of Econometrics 67, 748-756.

Sen, K. (1986), 'The Gini coefficenst and Poverty Indexes: Some Reconciliations', Journal of American Statistical Association 81, 1050-1057.

Theil, H. (1967), Economics and Information Theory, Amsterdam, North-Holland. 